http://jmscr.igmpublication.org/home/ ISSN (e)-2347-176x ISSN (p) 2455-0450 crossref DOI: https://dx.doi.org/10.18535/jmscr/v7i8.95

\title{
Clinical Presentation and Treatment Guidelines for Diabetic Foot Infections in Kanyakumari District
}

\author{
Authors \\ Dr Angeline Vincent ${ }^{1}$, Dr Senthil Kumar ${ }^{2}$ \\ ${ }^{1}$ Assistant Professor, ${ }^{2}$ Associate Professor, \\ Department of Surgery, Kanyakumari Government Medical College Hospital \\ *Corresponding Author \\ Dr Senthil Kumar \\ MS Surgery, Department of Surgery, Kanyakumari Government Medical College Hospital
}

\begin{abstract}
A diabetic foot is defined as any infra malleolar infection in a person with diabetes mellitus. The morbidity of this condition and its rising incidence has implications in the management. A multidisciplinary approach by a team of physician, surgeon, social care worker, a physiotherapist, a dietician and a podiatrist is necessary. This study over a period of 3 years includes 50 patients. $90 \%$ of our patients were above 30 years. About $40 \%$ were PEDIS grade 1 or 2 i.e with mild or no infections. About $22 \%$ had PEDIS grade 4 infections, $30 \%$ had evidence of peripheral vascular disease, neurological features were seen in $24 \%$ and renal failure in $20 \%$ of our patients. $65 \%$ of our patients had inframalleolar disease whereas the rest had leg or thigh involvement. Bone was involved in 3\% of our patients. More than two micro organisms were isolated in 53\% of our patients. Bacterial resistance was a problem in hospitalized patients. More than 50\% of the patients needed debridement. About $21 \%$ of our patients needed partial thickness skin grafting. $30 \%$ of our patients needed amputation of toes/midtarsal amputation or below knee amputation. Treatment guidelines for these patients is given here below.
\end{abstract}

Keywords: Diabetic foot infections - DFI, Soft tissue infections, Amputation.

\section{Introduction}

In this study we attempt to review the clinical presentation of diabetic foot in Kanyakumari District and treat diabetic foot infections aggressively because of the morbidity associated with such infections. Study pattern involves the age group, limb involvement, clinical presentation, presence of systemic sepsis, peripheral neuropathy, peripheral vascular disease, bone involvement, type of bacterial isolates, type and number of debridement sessions needed, amputations and their types performed and types of skin cover given.

\section{Materials and Methods}

No of patients - 50 patients

Study group - Male \& Female patients 30 - 60 years

Study Hospital- Kanyakumari Government

Medical College Hospital

Study period - June 2015 - June 2018

A diabetic foot is defined as inframalleolar infection in a diabetic patient. Infection spreading above the malleous includes cellulitis, myositis, necrotizing, soft tissue infections, septic arthritis, tendonitis and osteomyelitis. Commonly 
infections begin as a malperforens foot ulcer resulting from

1) Peripheral sensory neuropathy i.e loss of sensation in foot and leg leading to un attended minor injuries.

2) Peripheral motor neuropathy leading to abnormal foot anatomy and biomechanics causing pressure points, callus formation, ulcers, clawing of toes.

3) Peripheral autonomic neuropathy leads to dry and cracked skin.

4) Neuro osteo arthropathic deformities eg. Charcots disease.

5) Vascular insufficiency

6) Hyper glycemia

All patients were assessed by

1) History of foot ulcers or foot problems

2) Examination for foot deformity/ ulcer/infection

3) Testing for neuropathy

4) Pulsations

5) Range of mobility of joints

6) Venous insufficiency.

Patients were scored on the basis of PEDIS scoring system.

PEDIS Grade (1) Uninfected wound

PEDIS Grade (2) Infection limited to skin, superficial subcutaneous

Tissue, no local complications or systemic illness.

Erythema $<2 \mathrm{~cm}$ around the ulcer.

PEDIS Grade (3) Moderate infection in a patient who is systemically well and metabolically stable. Cellulitis more than $2 \mathrm{~cm}$ around the ulcer, deep tissue abscess, gangrene involving muscle, tendon, joint or bone.

PEDIS Grade (4) Severe infection in patient with systemic toxicity or metabolic instability eg. Fever, chills, hypotension, confusion, vomiting, acidosis, azotemia.

\section{Treatment Guidelines}

PEDIS grade 1 and 2 - Outpatient treatment

PEDIS grade 3 and 4

1) Stabilize patient - Correct fluid and electrolyte abnormality, correct hyperosmolality, correct acidosis and azotemia, restore normoglycemia and prepare for amputation.

2) Broad spectrum antibiotics against gram positive and gram negative organisms as well as anaerobes.

3) Ulcer debridement, skin cover - primary or delayed closure, amputations and vascular grafting.

4) Ulcer dressings - Eusol solution, moist wound healing dressings, appropriate foot wear.

5) Adjunctive treatment - Vacuum drainage, Recombinant growth factor, hyperbaric oxygen, antimicrobial dressings.

6) Non healing ulcer more than 6 weeks Treat for osteomyelitis.

\section{Analysis of Data}

1) More than $90 \%$ of patients were 30 years and above

2) $40 \%$ were PEDIS grade 1 or 2 having mild or no infection

3) About $22 \%$ had PEDIS grade 4 infections

4) $30 \%$ had evidence of peripheral vascular disease

5) $24 \%$ had peripheral neuropathy

6) $20 \%$ had renal failure

7) $65 \%$ had inframalleolar disease, rest had leg or thigh involvement.

8) Bone involved in $3 \%$ of patients

9) More than 2 micro organisms were isolated in $53 \%$ of patients

10) More than $50 \%$ needed ulcer debridement

11) $21 \%$ of patients had partial thickness skin grafting done

12) $30 \%$ of patients needed amputation of toes/ray amputation/midtarsal amputation or below knee amputation

\section{Conclusion}

The prevalence of diabetes is increasing. Inadequate patient education and inappropriate treatment often lead to delayed presentation and life threatening morbidity in diabetic patients. 
Complications of diabetes involving lower limbs range from cellulitis to limb threatening ischemia.

Diabetes enhances the progression of atherosclerosis and leads to peripheral arterial disease. There is a $80 \%$ increased risk of cardiovascular disease in patients with coexisting diabetes and peripheral vascular disease. Treatment is individualized according to the PEDIS score grade 1,2,3,4 uninfected, mild, moderate and severe infection respectively.

Imaging studies is used to identify the extent of infection and guide further management.

\section{Treatment guidelines for patients with DFI in Kanyakumari District}

1) Use of off loading devices or foot wear to reduce pressure at high pressure points.

2) PEDIS grading for foot infection and treatment accordingly

3) Medical treatment of diabetes with insulin, control of other risk factors, patient education on appropriate prevention strategies and empirical antibiotics.

4) Empirical broad spectrum antibiotic cover can be initiated in patients with PEDIS grade 2-4 which is tailored according to target organism based on culture and sensitivity. Fluoroquinolones to cover gram positive and gram negative organisms, metronidazole to cover anaerobic activity. Ceftriaxone and vancomycin is warrented in the presence of infection with Pseudomonas and MRSA. Piperazillin tazobactum combinations, linezolid and Ertapenem have also be used in DFI. Moderate infection require 3 weeks of antibiotics, bone involvement requires 6 weeks of antibiotic therapy.

5) Surgical intervention for gas gangrene, necrotizing infections, abscess, gangrene, joint involvement or osteomyelitis. Wound debridement to reduce the septic load.

6) Limb salvage surgery or amputation is done in worsening clinical scenario. The key element for amputation or limb salvage is to ensure adequate blood supply to the stump, infection control, glycemic control and providing biomechanical stability.

7) In patients with critically ischemic limbs vascular intervention should be performed early. However debridement should not be delayed till vascular intervention is performed. Atheroselerosis, intimal thickening and calcifications compromise vascularity and are a potential threat for revascularization procedures. Endovascular procedures and distal bypass are the revascularization procedures with moderate results

8) Bone destruction is treated by bone resection and antibiotic therapy

9) Some patients may be treated non operatively in the presence of major co morbidities.

10) Life threatening infection is treated by amputation of that foot

11) Dressings for moist healing wounds. Normal saline soaked dressings are given to encourage would healing

12) Adjunctive treatments like hyperbaric oxygen, growth factors, maggot larvae or topical vacuum assisted closure may be tried in patients whose wound healing is slow.

\section{References}

1. Lipsky BA, Berendt AR, Cornia PB, Pile JC, Peters Ej, Amstrong DG, et al, Infectious Diseases Society of America. 2012 Infectious Diseases Society of America clinical practice guideline for the diagnosis and treatment of diabetic foot infections. Clin Infect Dis. 2012 Jun;54(12):e132-73

2. Newman AB, Siscovick DS, Manolio TA, et al. ankle-arm index as a marker of atherosclerosis in the cardiovascular health study. Cardiovascular heart study (CHS) 
Collaborative research group. Cirvulation. 1993;88(3):837-45

3. Gregg EW, Sorlie P, Paulose-Ram, et al. Prevalence of lower-extremity disease in the US adult population $\geq 40$ hears of age with and without diabetes: 1999-2000 national health and nutrition examination survey. Diabetes care.2004;27(7):1591-7

4. Lipsky BA. Empirical therapy for diabetic foot infections: are there clinical clues to guide antibiotic selection? Clin Microbiol infect.2007;13(4):351-3

5. Taylor SM, Kalbaugh CA, Blakhurst DW, et al. Preoperative clinical factors predict postoperative functional outcomes after major lower limb amputations: an analysis of 553 consecutive patients. J Vasc surg. 2005;42(2):227-35. 